\title{
Germanica
}

\section{Georges Perec in der Literaturlandschaft der DDR, oder: Die Leerstelle der Shoah in Poetik und Erinnerungspolitik}

Georges Perec dans le paysage littéraire de la RDA, ou: la Shoah comme blanc dans la poétique et la politique du souvenir Georges Perec in the Literary Landscape of the GDR, or: The Void of the Shoah in Poetics and the Politics of Remembrance

\section{Gloria Freitag}

\section{OpenEdition}

Journals

Édition électronique

URL : http://journals.openedition.org/germanica/3563

DOI : 10.4000/germanica.3563

ISSN : 2107-0784

\section{Éditeur}

Université de Lille

\section{Édition imprimée}

Date de publication : 30 décembre 2016

Pagination : 171-179

ISBN : 9782913857384

ISSN : 0984-2632

\section{Référence électronique}

Gloria Freitag, „Georges Perec in der Literaturlandschaft der DDR, oder: Die Leerstelle der Shoah in Poetik und Erinnerungspolitik", Germanica [Online], 59 | 2016, Online erschienen am: 30 Dezember 2018, abgerufen am 06 Januar 2021. URL: http://journals.openedition.org/germanica/3563 ; DOI: https://doi.org/10.4000/germanica.3563 


\title{
Georges Perec in der Literaturlandschaft der DDR, oder: Die Leerstelle der Shoah in Poetik und Erinnerungspolitik
}

\author{
Gloria FrEITAG \\ Friedrich-Schiller-Universität Jena
}

Georges Perec ist in der Literaturlandschaft der DDR nicht leicht zu verorten. Das Verhältnis der Verlagsakteure zu diesem Autor changiert zwischen Interesse und Ablehnung: auf der einen Seite die Anerkennung der politischen Grundeinstellung des Autors; auf der anderen Seite die Distanzierung von einer Literatur, die sich in ihrem experimentellen Gestus selbst genüge - und dies seit dem Beitritt Perecs zur „Schreibwerkstatt“ Oulipo 1967. Nichtsdestotrotz gilt er im internationalen Programm des Verlags Volk und Welt als „Standardautor ““. Tatsächlich wurden drei seiner Werke veröffentlicht: 1965 erscheint der mit dem Prix Renaudot ausgezeichnete Roman Die Dinge, ein Jahr später die Novelle Was für ein kleines Moped...? und 1978 schließlich die autobiographische Erzählung $W$ oder die Erinnerung an die Kindheit. Dem stehen aber fünf seiner Bücher gegenüber, deren Veröffentlichung in einem sogenannten Druckgenehmigungsverfahren abgelehnt wurde ${ }^{2}$.

1. - Ariane Steiner, Georges Perec und Deutschland. Das Puzzle um die Leere, Würzburg, Königshausen \& Neumann, 2001, S. 250.

2. - Folgende Werke wurden in der DDR begutachtet (Jahreszahlen der 
Zumeist nimmt man Anstoß an Perecs „Sprachakrobatik[en] $]^{3 “}$, die ,auf

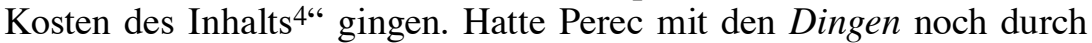
seine scharfsichtige Analyse von Gesellschaften überzeugt, die „keine im Sinne des Menschen ${ }^{5 "}$ seien, wurde seinen nachfolgenden Werken attestiert, sie gäben das Reale, Konkrete zugunsten eines rein ästhetischen Anspruchs preis.

Die Argumentationsstrategien in den Druckgenehmigungsakten verweisen auf bestimmte kultur- und geschichtspolitische Voraussetzungen: In der Veröffentlichungspraxis der DDR ist bekanntlich nicht in erster Linie die künstlerische Qualität der Texte ausschlaggebend, sondern deren politische „Grundorientierung6.“ Hinter der Literatur in der DDR steht die Literaturpolitik der DDR. Dennoch lässt sich die Veröffentlichungspraxis nicht auf die staatlich empfohlenen Auswahlkriterien reduzieren. Sie ist stattdessen weitaus komplexer und auch undurchsichtiger, insofern Verlage, Lektoren und Gutachter teilweise versuchten, ,problematische“ literarische Texte wertkonform darzustellen, durch Vorworte oder Nachworte zu ideologisieren oder in Anthologien zu verstecken ${ }^{7}$.

Die Rekonstruktion der Argumentationen, die ich im Folgenden vornehmen werde, dient auch zur Vorbereitung einer Analyse der Wertungskriterien, unter denen die Erzählung $W$ oder Erinnerung an die Kindheit wahrgenommen wird. Entscheidend für die Beurteilung dieses Textes ist vor allem der Erinnerungsdiskurs der DDR, der durch eine Marginalisierung der Ermordung der europäischen Juden geprägt wird ${ }^{8}$. Deren Verdrängung aus dem öffentlichen Gedenken wird bereits 1945 vorbereitet und 1953 u.a. durch die Gründung des „Komitees der

Begutachtungen in Klammern): Un homme qui dort (1965), La Disparition (1969), La Boutique Obscure (1975), La Vie mode d'emploi (1979; 1984 wird eine Veröffentlichung empfohlen, die allerdings nicht realisiert wird).

3. - Verlagsgutachten zu La Disparition (1969), unveröffentlicht, zitiert nach Steiner, Perec und Deutschland, a.a.O., S. 251.

4. - Gutachter-Aktennotiz zu La Boutique Obscure (1975), unveröffentlicht, zitiert nach Steiner, Perec und Deutschland, a.a.O., S. 252.

5. - Klaus Möckel, Verlagsgutachten zu Die Dinge (1966), Bundesarchiv DR 1 (Ministerium für Kultur) / 2333, Angaben im Folgenden in Kurzform.

6. - Vgl. Hans-Jürgen Hartmann, ,Französische Literatur in der DDR. Ein bibliographischer Kommentar“, in: Dorothee Röseberg (Hrsg.), Frankreich und „Das andere Deutschland." Analysen und Zeitzeugnisse, Tübingen, Stauffenberg-Verlag, 1999, S. 217-224, hier S. $220 \mathrm{f}$.

7. - Vgl. Siegfried Lokatis, „Die Hauptverwaltung des Leselandes“, APuZ, 11 (2009), S. 23-31, hier S. 25.

8. - Vgl. Wolfgang Emmerich, „Kein Holocaust? Die gekappte Darstellung des Zweiten Weltkriegs in der DDR-Literatur“, in: Jürgen Egyptien (Hrsg.), Erinnerung in Text und Bild. Zur Darstellbarkeit von Krieg und Holocaust im literarischen und filmischen Schaffen in Deutschland und Polen, Berlin, Akademie-Verlag, 2002, S. 17-31. 
Antifaschistischen Widerstandskämpfer" institutionalisiert, das keine

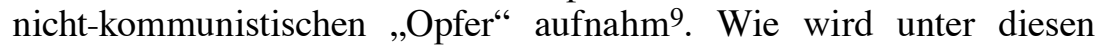
Prämissen eine Erzählung wahrgenommen, die in ihrem elaborierten Konstruktionsprinzip den Versuch unternimmt, die Leerstelle in die literarische Form einzutragen, die das Verschwinden eines einzigen Menschen hinterlässt, das demjenigen vieler, wenn nicht zahlloser Anderer gleicht? Der ,kollektiven Schicksallosigkeit“, die ein zentrales Merkmal des Ereignisses selbst ist, stellt Perec durch die Verfahren seiner Darstellung den Anspruch auf ein individuelles Schicksal gegenüber.

\section{Georges Perec in der Begutachtung}

Die Veröffentlichungen von Perec fallen in die Phase einer Neuorientierung. Während bereits in der SBZ fast ausschließlich französische Titel aus der Vorkriegszeit veröffentlicht wurden ${ }^{10}$, erweitert sich das Programm Anfang der 1960er Jahre um literarische Texte, die zur zeitgenössischen, ,gute[n], allgemeine[n], humanistische[n] ${ }^{11 " ~ L i t e r a t u r ~}$ gezählt werden: „Nach eher mageren Jahren der Veröffentlichung moderner, ausländischer Literatur war sich das gesamte Lektorat darin einig, dem DDR-Leser das Bestmögliche aus aller Welt zu liefern ${ }^{12 “,}$ so der Romanist Klaus Möckel. Zu den Errungenschaften der „,vorsichtigen literaturpolitischen Öffnung13“" gehört beispielsweise die Veröffentlichung einer Auswahl aus Kafkas Werk im Herbst $1965^{14}$. Dass sich im Dezember desselben Jahres infolge des 11. Plenums des ZK repressive Maßnahmen gegenüber Autoren verschärfen und gegenüber Verlagen die „Verantwortung“ angemahnt wird, die „Kulturpolitik der Partei 15" umzusetzen, kann als Folge eines zweifachen kulturpolitischen Anspruchs angesehen werden: Einerseits wird die Literatur

9. - Harry Waibel, Der gescheiterte Anti-Faschismus der SED. Rassismus in der $D D R$, Frankfurt a.M., Peter Lang, 2014, S. 64 f.

10. - Vgl. Hartmann, Französische Literatur, a.a.O., S. 218 ff.

11. - Klaus Möckel: Vom Kleinen Prinzen bis zum Zimmer der Träume, in: Simone Barck. Siegfried Lokatis (Hrsg.), Fenster zur Welt. Die Geschichte des DDR-Verlags Volk \& Welt, Berlin, Links, 2005, S. 125-132, hier S. 126.

12. - Vgl. ebd., S. 125.

13. - Siegfried Lokatis: Die Hauptverwaltung Verlage und Buchhandel, in: Simone Barck, Martina Langermann, Siegfried Lokatis (Hrsg.), ,Jedes Buch ein Abenteuer “. Zensur-System und literarische Öffentlichkeiten in der DDR bis Ende der sechziger Jahre, Berlin, Akademie-Verlag, 1997, S. 173-227, hier S. 220.

14. - Vgl. Michael Westdickenberg, Die »Diktatur des anständigen Buches«. Das Zensursystem der DDR für belletristische Prosaliteratur in den sechziger Jahren, Wiesbaden, Harrassowitz Verlag, 2004, S. 130 f.

15. - »Konzeption für Mitgliederversammlungen in Verlagen « vom Sektor Verlage, Buchhandel und wissenschaftliche Bibliotheken der Abteilung Wissenschaften des ZK der SED vom 14. Dezember 1965, zitiert nach Westdickenberg, Diktatur, a.a.O., S. 294. 
funktional als Instrument einer ideologisch vorgegebenen Erziehung begriffen, andererseits jedoch der Anspruch erhoben, ein literarisches Leben zu kultivieren, das dem internationalem Niveau entspricht ${ }^{16}$. Auch im Fall Kafka treffen diese beiden kaum zu vereinbarenden Ansprüche aufeinander: Die internationale Diskussion überführt die Marginalisierungsstrategie ihrer Haltlosigkeit und fordert eine differenziertere Auseinandersetzung mit dem zuvor reflexhaft als „dekadent" denunzierten Autor. Zusammenfassend lässt sich sagen, dass das 11. Plenum die Tendenzen einer Neubewertung und Akzeptanz moderner Gestaltungsmittel zwar zu „korrigier[en]“ sucht, jedoch nicht rückgängig machen kann ${ }^{17}$. Diese heterogenen Entwicklungen führen zu einer Konstellation, in der die internationale Gegenwartsliteratur ein neues Interesse erfährt, obwohl ihre Gestaltungsmittel weiterhin daraufhin befragt werden, inwiefern sie dem primären - d.h. erzieherischen - Anspruch an die Literatur genügen können. Diese Konstellation bildet auch den Hintergrund, vor dem Perecs Werke begutachtet werden.

In den Gutachten zu den Texten Die Dinge ${ }^{18}$ und Was für ein kleines Moped.... ${ }^{19}$ lassen sich mindestens fünf Kriterien voneinander unterscheiden, die zugunsten Perecs ausfallen: Wenn das „Repräsentativkriterium ${ }^{20 “}$ (1.) vorsieht, die bedeutendsten Texte der französischen Gegenwartsliteratur aufzunehmen, dann spricht die Preisauszeichnung des Romans Die Dinge für seine Veröffentlichung in der DDR. Das Auswahlkriterium Wirklichkeit (2.) erfüllt Perec in den Dingen durch seine Kapitalismuskritik und in Was für ein kleines Moped...? mit seiner Kritik am Algerienkrieg. Die unverkennbare Ablehnung des Krieges lasse auf die humanistische, antiimperialistische - wenngleich ideologisch noch unreife ${ }^{21}$ - Haltung (3.) des Autors schließen: Er steht „uns politisch nahe“22, urteilt Möckel. In formaler Hinsicht wird der dokumentarische Stil und die analytisch-diagnostische Kraft (4.) gewürdigt. Ausschlaggebend für die Veröffentlichung ist nicht zuletzt (5.) die ideologische sowie pädagogische Instrumentalisierbarkeit der Texte: „Ich brauche vielleicht nicht zu betonen, daß ein Wunschdenken, wie er es hier vorführt, auch bei

16. - Vgl. ebd., S. 186.

17. - Ebd., S. 130.

18. - Begutachtet von Klaus Möckel und Manfred Naumann.

19. - Begutachtet von Klaus Möckel und Henryk Keisch, der die Übersetzungen zu Die Dinge und Was für ein kleines Moped angefertigt hat.

20. - Vgl. Steiner, Perec und Deutschland, a.a.O., S. 250.

21. - Henryk Keisch, Gutachten zu Was für ein kleines Moped (1966), BArch DR $1 / 2333$.

22. - Klaus Möckel, Verlagsgutachten zu Was für ein kleines Moped, 27.10.1966, BArch DR $1 / 2333$. 
uns schon wieder ziemlich verbreitet ist. [...] Les choses ist kein großes Buch, aber es ist für uns nützlich ${ }^{23 . “}$

Gleichwohl wird bereits in dieser Zeit moniert, Perec belasse es bei einer Bestandsaufnahme. „Entscheidend“ sei jedoch „die Überwindung, nicht die detaillierte Schilderung negativer Erscheinungen 24", in deren Richtung die Literatur Wege aufzuzeigen habe, um die ihr zugeschriebene politisch-pädagogische Verantwortung zu erfüllen. Vor diesem Hintergrund erklärt sich die Perec attestierte „,ideologische Unreife“, die jedoch in zweifacher Hinsicht nutzbar gemacht wird: Einerseits wird mit den beiden Perec-Publikationen das Bedürfnis des DDR-Lesers nach französischer Gegenwartsliteratur befriedigt. Andererseits lässt sich der Vorrang der sozialistischen Literatur gegenüber der modernen Literatur zur Geltung bringen. Das Außengutachten von Manfred Naumann schließt dann auch mit der Einschätzung, das Buch sei repräsentativ für die ,relative Progressivität der französischen Gegenwartsliteratur ${ }^{25}$ “ - mitzulesen ist: gemessen am Maßstab der Progressivität der sozialistischen Literatur.

\section{Das Bild des Autors im Lichte der NS-Gedächtnisbildung $\operatorname{der} D D R$}

Neben den Druckgenehmigungsverfahren selbst sind die biographischen Skizzen aufschlussreich für eine Rekonstruktion des ostdeutschen Blicks auf Perec. Dies gilt beispielsweise für die Darstellung, die Frauke Rother in der Anthologie Französische Erzähler aus sieben Jahrzehnten (1985) vornimmt, in die Perec - neben anderen ,problematischen“, „bürgerlichen“ Autoren wie Gide, Céline, Duras, de Beauvoir - wiederaufgenommen wird. In ihrem Nachwort unternimmt Rother den Versuch, die Entwicklung der französischen Literatur seit der Jahrhundertwende nachzuzeichnen und in ihrem Verhältnis zur nationalen sowie international relevanten Geschichte herauszuarbeiten. Die in formaler sowie gattungstheoretischer Hinsicht heterogenen Nachkriegsströmungen nouveau roman, Existentialismus und Sprachspiel ließen sich unter inhaltlichen Gesichtspunkten in eine gemeinsame Perspektive rücken: „Die Auseinandersetzung mit Krieg, Faschismus, Okkupation, Resistance und in wachsendem Maße mit der Kollaboration nimmt in der Literatur bis zum heutigen Zeitpunkt einen auffallend großen Platz ein. Selbst jüngere Autoren, die diese historische Realität nicht oder nur mittelbar erfahren haben, greifen die damit

23. - Klaus Möckel, Verlagsgutachten zu Die Dinge (1966), BArch DR 1 / 2333.

24. - »Konzeption für Mitgliederversammlungen in Verlagen«, zitiert nach Westdickenberg, Diktatur, a.a.O., S. 294 ff. (Anhang), hier S. 294.

25. - Manfred Naumann, Gutachten zu Die Dinge (1966), BArch DR 1 / 2333. 
verbundenen, nach wie vor aktuellen Fragen in ihren Werken auf, so [...] Georges Perec in der Erzählung $W$ oder die Erinnerung an die Kindheit ${ }^{26 . " ~ I n ~ d e r ~ K u r z b i o g r a p h i e ~ i m ~ g l e i c h e n ~ B a n d ~ h e i ß t ~ e s: ~ „ 1936 ~}$ in Paris geboren. Romanschriftsteller, Erzähler. Jüdisch-polnischer Herkunft, Sohn eines Arbeiters. Verliert früh seine Eltern, sein Vater fällt im ersten Weltkrieg, seine Mutter kommt im KZ um. [...] ${ }^{27 “ .}$

Der Zweite Weltkrieg und die Okkupation Frankreichs bilden zweifellos den realgeschichtlichen Rahmen der Erzählung. Diese Aspekte zugleich auch als thematische Schwerpunkte zu verstehen, wäre jedoch eine Vereinfachung. Das gilt auch für den Zentralbegriff „Faschismus”, denn es geht Perec weniger um eine Analyse des Faschismus im Geflecht seiner sozioökonomischen und politischen Ursachen ${ }^{28}$, sondern darum, das Zerbrechen von Leben und Lebensgeschichten in einem allesumgreifenden System der „Vernichtung” zur Darstellung zu bringen - in einer Form, die nicht die schon stehenden Begriffe und Symbole wiederholt, mit denen die Konzentrations- und Vernichtungslager aufgerufen werden.

Die oben zitierte Charakterisierung und Kategorisierung des Autors ist symptomatisch für die DDR-Geschichtspolitik und ihre geschichtsphilosophischen Grundlagen. Zum Kernbestand dieser Politik gehört der Anspruch, sich im Bild des kommunistischen Widerstandskämpfers der geschichtlichen Handlungsfähigkeit zu vergewissern und diese für Gegenwart und Zukunft durch den Aufbau und die Stabilisierung der sozialistischen Gesellschaft zu sichern ${ }^{29}$. Die Kontinuierung eines bereits zur NS-Zeit „erbrachte[n] Antifaschismus 30“ entlastet nicht nur von einer selbstkritischen Auseinandersetzung mit der nationalsozialistischen Vergangenheit, sie verunmöglicht gleichsam apriori die Anerkennung derjenigen Gruppen, die nicht aufgrund eines regimekritischen und weltanschaulich orientierten Engagements, sondern aufgrund ihrer „rassischen“, religiösen, sexuellen oder sozialen Zugehörigkeit verfolgt und ermordet wurden. Ein weiteres Symptom dieser geschichtspolitischen Weichenstellung ist die mangelnde Sensibilität für die Differenzierung zwischen Konzentrations- und Vernichtungslagern

26. - Frauke Rother, ,Geschichte und Geschichten in der neuen französischen Prosa,“ in: Klaus Möckel, Frauke Rother (Hrsg.), Französische Erzähler aus sieben Jahrzehnten, Berlin, Volk und Welt, 1985, Nachwort, S. 654-687, hier S. 680 f.

27. - Ebd., S. $694 \mathrm{f}$.

28. - Vgl. Judith Klein, Literatur und Genozid. Darstellungen der nationalsozialistischen Massenvernichtung in der französischen Literatur, Wien/ Köln/ Weimar, Böhlau, 1992, S. 150.

29. - Vgl. Westdickenberg, Diktatur, a.a.O., S. $166 \mathrm{f}$.

30. - Harald Schmid, Antifaschismus und Judenverfolgung. Die »Reichskristallnacht « als politischer Gedenktag in der DDR, Göttingen, V \& R Unipress, 2004, S. 13. 
und eine Privilegierung ersterer als Handlungsorte kommunistischen Widerstandes. Die Leerstelle, welche die Vernichtungslager als Stätten der Shoah jahrzehntelang in der offiziellen DDR-Erinnerung bildet ${ }^{31}$, hat sich auch in die Beurteilung der Erzählung $W$ eingeprägt.

\section{Die Leerstellen der Shoah}

Einstimmig hervorgehoben wird in den Gutachten, dass Perec ,erstmals wieder ein bedeutsames Thema“ aufgreife und an die ,gesellschaftskritische Absicht ${ }^{32}$ " seines ersten Romans anknüpfe. Obzwar Perec dem Realitätskriterium genüge, zeige sich an der Konstruktion seines Werkes, dass er der Thematik nicht gewachsen sei und „noch immer herumexperimentiert ${ }^{33}$." Diese Einschätzung ist eine Folge davon, dass die Gutachter in der Darstellung eines „nach faschistischem Muster organisierten Staatswesens ${ }^{34 " ~}$ die Thematik des Textes erkennen. Diese thematische Setzung bildet den Ansatzpunkt einer einstimmigen Kritik: Die Sportinsel $W$, in der Rother ein Symbol für „,die Welt der faschistischen KZ's ${ }^{35 \text { “ }}$ erkennt, werde so beschrieben, ,,als ob sowohl die Grausamkeit der Machthabenden als auch die Hörigkeit der Unterworfenen unwandelbare, durch nichts hervorgerufene und durch nichts $\mathrm{zu}$ beeinflussende Voraussetzungen seien, von denen ausgehend sich die Vorgänge dann nur noch mechanisch abspielen ${ }^{36}$.“ Mit der Beschreibung einer ins Totale ausgreifenden Herrschaft begeht Perec geradezu einen faux pas, indem er durch die Grenzverwischung zwischen Tätern und Opfern am Bild des politischen Inhaftierten rüttelt. Sie wird wohl auch deshalb als unzureichend empfunden, weil

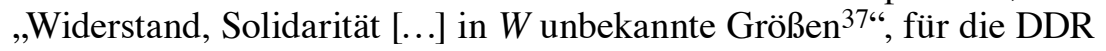
allerdings, und zwar in ihrer dezidiert antifaschistischen Lesart, staatstragend sind.

Die Kritik lautet ferner, Perec gehe in der Beschreibung - und dies sei dem symbolreichen Konstruktionsprinzip seiner Darstellung geschuldet - nicht weit genug, es fehle ihm eine sozioökonomische Perspektive: „Ein umfassendes Bild des Faschismus, seiner Entstehung

31. - Vgl. ebd., S. 16.

32. - Frauke Rother, Verlagsgutachten zu W oder die Erinnerung an die Kindheit (1977), BArch DR $1 / 2368$.

33. - Klaus Möckel, Gutachten zu W oder die Erinnerung an die Kindheit (1977), BArch DR 1 / 2368.

34. - Rother, BArch DR $1 / 2368$.

35. - Rother, BArch DR $1 / 2368$.

36. - Gutachten zu W oder die Erinnerung an die Kindheit (1975), in: Danielle Risterucci-Roudnicky: Nausikaa. La réception de la littérature française en RDA (19451990), Éditions de la Maison des sciences de l'homme, Paris, 2010.

37. - Klein, Literatur und Genozid, a.a.O., S. 151. 
und jener Faktoren, die zu seiner Vernichtung führen 38 ", würde nicht vermittelt, doch denunziere Perec „,wesentliche Merkmale faschistischer Herrschafts- und Unterdrückungsmechanismen ${ }^{39 “}$ - ein Indikator für die ideologische „Sauberkeit“ des Buches.

In der Kritik wird der Text an einem Anspruch gemessen, der sich aus der Geschichtspolitik der DDR, nicht aber aus der Erzählung selbst ergibt. In den Gutachten bildet sich die Leerstelle ab, die die Shoah in der Erinnerungspolitik der DDR darstellt, sowie die damit einhergehende Marginalisierung des „sinnlosen Leiden““ (Emmanuel Lévinas) derjenigen, die nicht als „,antifaschistische“ Kämpfer „gefallen“ sind. So avanciert das Verschwinden von Perecs Mutter im Verlagsgutachten von Klaus Möckel zu einem Sympathiekriterium: „Der Vater des Autors fiel zu Beginn des Krieges, die Mutter wurde nach Auschwitz gebracht und dort ermordet - diese Tatsachen rühren an und nehmen den Leser sofort für Perec ein 40 .“

Die Würdigung der Leerstelle(n), die Perec mithilfe von Zeichen als Präsenz $\mathrm{z}^{41}$ in den Text einträgt, als Elemente der Rührung ist in ästhetischer, ethischer sowie in politischer Hinsicht fragwürdig. Eine solche Einschätzung ergibt sich aus einer Perspektive, die den Nationalsozialismus reduktionistisch ausschließlich als Widersacher des Sozialismus und vor allem das Gedenken jener Opfer der Konzentrationslager hervorhebt, die zur Gruppe der kommunistischen Widerstandskämpfer gehören. In der Konsequenz ergibt sich daraus die Sicht auf Juden als ,,minder angesehene Opfer" ${ }^{\prime 2}$ des Faschismus.

Die Erzählung stellt sich demgegenüber in ihren starken selbstreferentiellen Passagen ihrem eigenen Scheitern an den Ereignissen und hebt das, was durch die Benennung nur verfehlt werden kann, in einem Gestus des Zeigens auf. Das Leiden in individueller Perspektive wird vom kollektiven Charakter des Ereignisses selbst entwertet. Die Frage, die die autobiographische Geschichte eröffnet und durchzieht, lautet: Wie lässt sich die Leere innerhalb der eigenen Lebensgeschichte erzählen, wenn dieser stets schon , die große Geschichte, die Welthistorie mit dem großen Hackebeil43“" zuvorkommt und mit einer Statistik antwortet, in der das Verschwinden einer Einzigen nur eines ist unter sechs Millionen? Perec bildet das Verschwinden seiner Mutter im Text als Leerstelle(n) ab: zwischen dem ersten und zweiten Teil der Erzählung,

38. - Rother, BArch DR $1 / 2368$.

39. - Rother, BArch DR $1 / 2368$.

40. - Möckel, BArch DR $1 / 2368$.

41. - Klein, Literatur und Genozid, a.a.O., S. 154.

42. - Waibel, Anti-Faschismus, a.a.O., S. 64.

43. - Georges Perec, $W$ oder die Erinnerung an die Kindheit, aus dem Französischen von Thorgerd Schücker. Berlin, Volk und Welt, 1978, S. 12. 
zwischen der autobiographischen Erzählung vor und der Erzählung nach dem Verschwinden der Mutter, ist eine leere Seite eingefügt. Was sich darauf befindet, ist das Zeichen einer gleichsam in der großen, politischen Geschichte eingeschlossenen Leere: ,[...]“.

Die genannten Gutachten werfen ein Licht auf die Wahrnehmung der Erscheinungsweisen des Fehlens in weiteren, in der DDR abgelehnten Texten von Perec. Die Ablehnungen richten sich gegen akrobatische Formprinzipien und verkennen die reflektierten Darstellungen der Auslassung, mit denen Perec realgeschichtliche Erfahrungen des Fehlens ins Werk setzt, indem er beispielsweise romanlang den Vokal $e$ auslässt, der wichtigste Vokal in der französischen Sprache. Die Erzählung $W$ ist dieser Leerstelle gewidmet. Die Widmung lautet schlicht: „Für E“. Auf die Negativität der Shoah-Erfahrung antwortet Perec mit einer Poetik der Leerstelle. Indem die Darstellungsverfahren der Moderne abgelehnt werden, wird eine bestimmte Erinnerungskonzeption ausgeschlossen, die Erfahrungen in ihrer Individualität zu denken versucht und dabei ihr eigenes Scheitern an der Spannung zwischen der kollektiven Dimension des Ereignisses und dem Anspruch auf Einzigartigkeit der Erfahrung vorführt. 
